\title{
Radioembolization in the Treatment of Neuroendocrine Tumor Metastases to the Liver
}

\author{
Martin Vyleta and Douglas Coldwell \\ Department of Radiology, University of Louisville School of Medicine, Louisville, KY 40202, USA \\ Correspondence should be addressed to Martin Vyleta, msvyle01@louisville.edu
}

Received 11 July 2011; Accepted 1 November 2011

Academic Editor: Dan Granberg

Copyright ( $) 2011$ M. Vyleta and D. Coldwell. This is an open access article distributed under the Creative Commons Attribution License, which permits unrestricted use, distribution, and reproduction in any medium, provided the original work is properly cited.

\begin{abstract}
Surgical excision remains the preferred treatment for resectable hepatic metastases of neuroendocrine tumors. In cases of more disseminated hepatic disease, transarterial radioembolization with Yttrium-90- (90Y-) labeled microspheres has been demonstrated as a viable option for symptom and locoregional tumor control. On an outpatient basis, radioembolization can be utilized from early line to salvage phases, in various combinations with systemic therapies. Review of available data shows encouraging safety and efficacy profiles for the intraarterial application of $90 \mathrm{Y}$ for the treatment of mNETs of the liver. Symptom control and decrease in somatostatin analog use can be achieved, as well as prolonged survival.
\end{abstract}

\section{Introduction}

After removal of the primary, isolated metastases of neuroendocrine tumors to the liver can be curatively approached by surgical resection with achievable disease-free survival of 42 to 46 months depending on the extent of metastases [1]. However, only $10 \%$ of metastatic liver disease cases are resectable [2]. Also, significant surgical morbidity and mortality as well as the unpredictable natural history of malignant neuroendocrine neoplasms has to be considered [2]. Consensus guidelines for digestive (neuro)endocrine tumors currently call for complete hepatic deposit resection or $90 \%$ debulking if feasible [3] since their introduction in the 1980s somatostatin analogs have significantly helped in symptom control of functional neuroendocrine tumors. Their effectiveness, however, tends to diminish over time due to tachyphylaxis and disease progression. An antiproliferative effect has been ascribed to somatostatin analogs on their own [4]. Additional labeling with radionuclides (e.g., 177Lutetium-DOTA-TATE) has shown response rates of 13$30 \%$ and median overall survival of 13-46 months along with significant clinical benefit [5-10].

Overall, metastatic neuroendocrine tumors have exhibited modest response to systemic chemotherapy. Streptozocin- and doxorubicin-based protocols achieve response rates of up to $16 \%$. In more poorly differentiated NETs a $41.5 \%$ response rate has been seen with cisplatin/etoposide combinations [6-10]. Recently, 70\% partial remissions with estimated overall survival of $92 \%$ at 2 years have been reported with a temozolamide/capecitabine regimen in metastatic NET of the pancreas [11].

Novel agents targeting diverse receptors are in various stages of development. Preliminary data for sunitinib, a multikinase inhibitor, for example, demonstrate a promising progression-free survival of 11.4 months versus 5.5 months for placebo.

\section{Transarterial (CHEMO) Embolization (TA $[\mathbf{C}] \mathbf{E})$}

Retrospective studies have shown benefit to both TAE and TACE in the treatment of mNET with regard to tumor growth, symptom control, and biochemical surrogates. In the most recent large retrospective study of 123 treated patients undergoing an average of 7 chemoembolization cycles each, 62\% partial response was seen with overall 3-, 5 -, and 10-year survivals of 59, 36, and 20\%, respectively, and overall mean survival of 5.47 years [12]. 
This result is corroborated by the 67\% ORR (mean survival 33.8 months) in another large study employing TACE and TAE for the treatment of carcinoid hepatic metastases. A separate group of islet cell carcinomas in the latter study exhibited an ORR of only 34\% (mean survival 23.2 months); in the former study 10 cases with insulin/glucagon secretion were subsumed in the study population $[12,13]$.

No consensus has been established regarding technique, embolizing, and chemotherapeutic agents in TACE. Overall, both TAE and TACE appear to elicit similar responses from NET liver metastases, suggesting relative primacy of the embolic effect $[2,14]$.

\section{Transarterial Radiombolization (TARE)}

Transarterial radioembolization (TARE) or selective internal radiotherapy (SIRT) with Yttrium-90 microspheres represents a further viable option in the treatment arsenal for nonresectable liver metastases of both functional and nonfunctional NETs. A number of retrospective as well as a few prospective studies demonstrate efficacy and safety combined with the convenience of an outpatient procedure that rarely requires hospitalization (Table 1) [15-25]. What is more, it appears that the primarily one-time treatments with TARE compare favorably to usually multiple procedures required $\mathrm{TA}(\mathrm{C}) \mathrm{E}$ regimens.

Two kinds of Yttrium-90 microspheres are currently approved for treatment of unresectable liver tumors on the European market: Yttrium-90 resin microspheres (SIRspheres; Sirtex Medical, Sydney) with 20-60 $\mu \mathrm{m}$ diameter mounted with a radioactive load of approximately $50 \mathrm{~Bq}$ per sphere, as well as 20-30 $\mu \mathrm{m}$ glass microspheres (TheraSphere, MDS Nordion, Ottawa) with the higher radioactive load of $2500 \mathrm{~Bq}$. SIR-spheres come with premarket approval (PMA) for unresectable hepatic metastases of colorectal cancer in the United States and may be utilized "off-label" for other tumors without prejudice under the using physicians discretion authority and responsibility. Therasphere has a humanitarian device exception for the treatment of hepatocellular carcinoma in the US which is more restrictive than PMA and requires an IRB protocol and limitations to hepatocellular carcinoma (HCC) only.

A phase II trial of Yttrium-90 resin microspheres in combination with systemic FU chemotherapy was conducted with 34 patients. While this represented first-line therapy for the majority of patients, $29 \%$ had failed prior liver resection and $15 \%$ had received earlier chemotherapy, with 59\% also manifesting extrahepatic disease. Complete response was effected in $18 \%$, partial response in $32 \%$, and stable disease $15 \%$ of cases. Symptom improvement and Chromogranin A decrease at 6 months were seen in $50 \%$ and $41 \%$ of cases, respectively. $59 \%$ of patients remained alive at $37 \pm 2$ months, with 12 patients without hepatic recurrence at 33.3 \pm 2.3 months $[20]$.

Another prospective trial with Yttrium-90 resin microspheres involved ten patients with progressive or symptomatic unresectable hepatic spread of NET. $40 \%$ partial response was seen at 6 months and 2 out of 3 patients experienced symptomatic improvement, and average quality of life climbed to general-population levels by 6 months. Seven patients were still alive at 35 months followup, with the three intervening deaths attributable to progression of extrahepatic disease. Little toxicity was observed, with the authors noting comparable to better toleration of TARE compared to TACE [23].

Another study with Yttrium-90 resin microspheres also had quality of life as an endpoint and could demonstrate significant improvement of quality of life [18].

In a third prospective trial, 20 and 22 patients with mNET were treated with resin and glass Yttrium-90 microspheres, respectively, after the patient had previously undergone surgery (36\%), ablation (19\%), TA(C)E (14\%), and symptomatic treatment $(60 \%)$. ORR resulted in $50 \%$ and $54 \%$, and stable disease in $44 \%$ and $38.5 \%$, respectively. Projected median survival times were 28 and 22 months, respectively (not statistically significant difference), with the majority of patients alive at time of study publication [22].

The largest retrospective study to date reviewed a total of 148 patients in 10 clinical centers treated with Yttrium90 resin microspheres in a primarily salvage setting of mNET. 70 months median survival with 63\% ORR and 23\% stable disease were demonstrated, with most deaths due to progression of extrahepatic disease. Toxicity was very low, no radiation-induced liver disease (RILD) was seen even in the 33 patients receiving retreatment of the same liver lobe(s) [19].

Repeated radioembolizations of both or single lobes were also performed in a minority of patients in other studies, with a few receiving a third treatment $[19,20,24,25]$. No case of RILD was seen and an increased duration of tumor response in cases of hepatic $\mathrm{mNET}$ progression was recognized $[18,19,24,25]$.

\section{Discussion}

Overall, the available studies demonstrate effective and safe use of radioembolization for liver dominant disease in mNET. There is a positive effect on symptoms, quality of life, response criteria, and survival in various stages of progression, which is mirrored in the recommendations of the Radioembolization Brachytherapy Oncology Consortium [26]. A robust safety profile for radioembolization with Yttrium-90 microspheres was confirmed in two 2009 analyses $[26,27]$. The overall incidence of RILD, in particular, was estimated to be $0.8 \%$ [26].

Evidence-based data for treatment decisions in inoperable mNET are not presently available. A recent overview of strategies for advanced enteropancreatic NET comprehensively presents the panorama of current and developing multimodal treatment options. In general, former waitandsee strategies even in well-differentiated tumors with minor-to-moderate tumor loads will have to be reevaluated, as tumor progression, with median TTP of 6 months, is inevitable [5].

In order to relieve the common carcinoid syndrome consisting of diarrhea, flushing, hypertension, bronchoconstriction, right valvular heart failure and other endocrine effects, or effect medication sparing, at least palliative tumor 
TABLE 1: Study outcomes of TARE for NET liver metastases.

\begin{tabular}{|c|c|c|c|c|c|c|c|}
\hline Lead Author, Year & $n$ & Prospective? & Treatment Phase & ORR & $\mathrm{SD}$ & $\begin{array}{l}\text { Symptom } \\
\text { Response }\end{array}$ & Survival \\
\hline Arslan, 2011 [17] & 10 & No & $?$ & $80 \%$ & $10 \%$ & NR & All alive at $4-28 \mathrm{mo}$ \\
\hline Cao, $2010[15]$ & 51 & No & $?$ & $38 \%$ & $27 \%$ & NR & $36 \mathrm{mo}$ \\
\hline Saxena, 2010 [26] & 48 & No & $?$ & $55 \%$ & $23 \%$ & NR & $35 \mathrm{mo}$ \\
\hline Kalinowski, 2009 [19] & 9 & Yes & Refractory & $66 \%$ & $33 \%$ & Improved QoL & $57 \%$ alive at $36 \mathrm{mo}$ \\
\hline Kennedy, 2009 [27] & 148 & No & Refractory/salvage & $63.2 \%$ & $22.7 \%$ & NR & $70 \mathrm{mo}$ \\
\hline King, 2008 [22] & 34 & Yes & First-line* & $50 \%$ & $14.7 \%$ & $50 \%$ & $\begin{array}{l}59 \% \text { alive at } 37 \pm \\
2 \mathrm{mo}\end{array}$ \\
\hline Murthy, 2008 [16] & 8 & No & Refractory/salvage & $12.5 \%$ & $50 \%$ & NR & $14 \mathrm{mo}$ \\
\hline Rhee, 2008 [23] & $\begin{array}{c}20 \\
22^{* *}\end{array}$ & $\begin{array}{l}\text { Yes } \\
\text { Yes }\end{array}$ & $\begin{array}{l}\text { Refractory } \\
\text { Refractory }\end{array}$ & $\begin{array}{c}50 \% \\
53.8 \%\end{array}$ & $\begin{array}{c}44 \% \\
38.5 \%\end{array}$ & $\begin{array}{l}\mathrm{NR} \\
\mathrm{NR}\end{array}$ & $\begin{array}{l}28 \mathrm{mo} \\
22 \mathrm{mo}\end{array}$ \\
\hline Meranze, 2007 [24] & 10 & Yes & First-line & $40 \%$ & $60 \%$ & Improved QoL & $70 \%$ alive at $35 \mathrm{mo}$ \\
\hline McGrath, 2007 [25] & 26 & No & First-line/refractory & $58.3 \%$ & $33 \%$ & NR & $69 \%$ alive at $17.3 \mathrm{mo}$ \\
\hline Kennedy, 2006 [21] & 18 & No & First-line/refractory & $89 \%$ & NR & NR & $89 \%$ alive at $27 \mathrm{mo}$ \\
\hline
\end{tabular}

* combined with 5-FU.

** with Yttrium-90 glass microspheres, all others with Yttrium-90 resin microspheres. Median survival in last column unless otherwise indicated. ORR: objective response rate (complete + partial response). SD: stable disease. NR: not reported. QoL: quality of life.

debulking, either surgically or with transarterial approaches, is necessary. Hepatic tumor burden debulking appears to improve survival as well [28-32]. 5-year survival rates for mNET are $50-59 \%$ according to national cancer registries $[33,34]$. With intervention, 5 -year survival reaches $72-100 \%$ in limited hepatic spread and $25-51 \%$ in more extensive hepatic spread $[35,36]$.

TARE (see Table 1) is at least comparable to TA(C)E in effectiveness $[13,14]$. An advantage of the TARE protocol is the generally single treatment to achieve this effect, while $\mathrm{TA}(\mathrm{C}) \mathrm{E}$ is generally applied multiple times. This obvious patient comfort and quality of life advantage is compounded by the well-known lower severity of the "postembolization" syndrome (which in TARE's case represents radiation irritation) and reduced need for inpatient admittance. On the other hand, preparatory angiography mapping, embolization, and treatment simulation are a necessary first step in TARE workup.

It is conceivable that Yttrium-90 microspheres can be held in reserve, so to speak, for further repeated use to establish prolonged disease control. In this respect, it seems superior to repeated $\mathrm{TA}(\mathrm{C}) \mathrm{E}$. For one thing further extended use of $\mathrm{TA}(\mathrm{C}) \mathrm{E}$ is limited by the sheer number of necessary repetitions, reducing patient days outside the hospital and thus quality of life. The extended use of TARE, on the other hand, would likely not reach the number of regular $\mathrm{TA}(\mathrm{C}) \mathrm{E}$ sessions. Similar advantages also apply to side effects and outpatient flexibility. Also, the degree of embolic effect could skew repeated treatment schemes to TARE's favor: while $\mathrm{TA}(\mathrm{C}) \mathrm{E}$ may "prune" the arterial tree to such an extent that agent delivery is impaired due to the larger particle size (100-300 $\mu \mathrm{m}$ versus $35 \mu \mathrm{m}$ for TARE), the lesser embolic effect of TARE may keep the path to the tumor more reliably open. Further examination of the limits of dose and repeat application with regard to RILD could possibly enable attempts at treating even more advanced hepatic spread with radioembolization. An investigation of radiosensitizers, for example, capecitabine, to be concurrently used with TARE may also be warranted as this could represent another lever to further potentiate its effects [37].

Apparently disease control through radioembolization treatment is effective and long-lasting enough for most patients in the observed time spans to succumb to progressive extrahepatic disease. Thus, initiation/resumption of chemotherapy, possibly combined with extrahepatic local resection/ablation if necessary, appears reasonable.

Further considerations concern the endpoints surveyed in Yttrium-90 treatment studies. Morphologic response criteria (i.e., RECIST) may be misleading in TARE followups. Since the RECIST criteria were designed to reflect the response to systemic chemotherapy, the response to TARE is not gradual shrinkage but the creation of a necrotic tumor whose margins may not be well circumscribed in the baseline CT yielding a reading of progressive disease or mere stable disease. Collection of tumor marker data, for example, Chromogranin A, as well as functional parameters, for example, PET, may help to overcome some of these shortcomings. Emphasis should also be laid on formal measurements of quality of life in subsequent studies of TARE to better gauge this essential patient dimension.

\section{Disclosure}

Dr. Coldwell is a consultant to Sirtex, Inc.

\section{References}

[1] K. A. Yao, M. S. Talamonti, A. Nemcek et al., "Indications and results of liver resection and hepatic chemoembolization for metastatic gastrointestinal neuroendocrine tumors," Surgery, vol. 130, no. 4, pp. 677-685, 2001.

[2] D. C. Madoff, S. Gupta, K. Ahrar, R. Murthy, and J. C. Yao, "Update on the management of neuroendocrine hepatic 
metastases," Journal of Vascular and Interventional Radiology, vol. 17, no. 8, pp. 1235-1250, 2006.

[3] T. Steinmuller, R. Kianmanesh, M. Falconi et al., "Consensus guidelines for the management of patients with liver metastases from digestive (neuro)endocrine tumors: foregut, midgut, hindgut, and unknown primary," Neuroendocrinology, vol. 87, no. 1, pp. 47-62, 2008.

[4] A. Rinke, H.-H. Müller, C. Schade-Brittinger et al., "Placebocontrolled, double-blind, prospective, randomized study on the effect of octreotide LAR in the control of tumor growth in patients with metastatic neuroendocrine midgut tumors: a report from the PROMID study group," Journal of Clinical Oncology, vol. 27, no. 28, pp. 4656-4663, 2009.

[5] C. J. Auernhammer and B. Göke, "Therapeutic strategies for advanced neuroendocrine carcinomas of jejunum/ileum and pancreatic origin," Gut, vol. 60, no. 7, pp. 1009-1021, 2011.

[6] C. Waldherr, M. Pless, H. R. Maecke et al., "Tumor response and clinical benefit in neuroendocrine tumors after $7.4 \mathrm{Gbq}$ 90Y-DOTATOC," Journal of Nuclear Medicine, vol. 43, no. 5, pp. 610-616, 2002.

[7] M. K. McStay, D. Maudgil, M. Williams et al., "Large-volume liver metastases from neuroendocrine tumors: hepatic intraarterial 90Y-DOTA-lanreotide as effective palliative therapy," Radiology, vol. 237, no. 2, pp. 718-726, 2005.

[8] R. Valkema, S. Pauwels, L. K. Kvols et al., "Survival and response after peptide receptor radionuclide therapy with $\left[{ }^{90} \mathrm{Y}-\right.$ DOTA $^{0}, \mathrm{Tyr}^{3}$ ] octreotide in patients with advanced gastroenteropancreatic neuroendocrine tumors," Seminars in Nuclear Medicine, vol. 36, no. 2, pp. 147-156, 2006.

[9] D. J. Kwekkeboom, W. W. de Herder, B. L. Kam et al., "Treatment with the radiolabeled somatostatin analog $\left[{ }^{177} \mathrm{Lu}-\right.$ DOTA $^{0}, \mathrm{Tyr}^{3}$ ] octreotate: toxicity, efficacy, and survival," Journal of Clinical Oncology, vol. 26, no. 13, pp. 2124-2130, 2008.

[10] E. S. Delpassand, J. Sims-Mourtada, H. Saso et al., "Safety and efficacy of radionuclide therapy with high-activity in111 pentetreotide in patients with progressive neuroendocrine tumors," Cancer Biotherapy and Radiopharmaceuticals, vol. 23, no. 3, pp. 292-300, 2008.

[11] E. Raymond, L. Dahan, J.-L. Raoul et al., "Sunitinib malate for the treatment of pancreatic neuroendocrine tumors," The New England Journal of Medicine, vol. 364, no. 6, pp. 501-513, 2011.

[12] J. R. Strosberg, R. L. Fine, J. Choi et al., "First-line chemotherapy with capecitabine and temozolomide in patients with metastatic pancreatic endocrine carcinomas," Cancer, vol. 117, no. 2, pp. 268-275, 2011.

[13] X. D. Dong and B. I. Carr, "Hepatic artery chemoembolization for the treatment of liver metastases from neuroendocrine tumors: a long-term follow-up in 123 patients," Medical Oncology. In press.

[14] S. Gupta, M. M. Johnson, R. Murthy et al., "Hepatic arterial embolization and chemoembolization for the treatment of patients with metastatic neuroendocrine tumors: variables affecting response rates and survival," Cancer, vol. 104, no. 8, pp. 1590-1602, 2005.

[15] C. Q. Cao, T. D. Yan, L. Bester, W. Liauw, and D. L. Morris, "Radioembolization with yttrium microspheres for neuroendocrine tumour liver metastases," British Journal of Surgery, vol. 97, no. 4, pp. 537-543, 2010.

[16] R. Murthy, P. Kamat, R. Nunez et al., "Yttrium-90 microsphere radioembolotherapy of hepatic metastatic neuroendocrine carcinomas after hepatic arterial embolization," Journal of Vascular and Interventional Radiology, vol. 19, no. 1, pp. 145151, 2008.
[17] N. Arslan, M. Emi, E. Alagöz et al., "Selective intraarterial radionuclide therapy with Yttrium-90 (Y-90) microspheres for hepatic neuroendocrine metastases: initial experience at a single center," Vojnosanitetski Pregled, vol. 68, no. 4, pp. 341$348,2011$.

[18] A. T. Ruutiainen, M. C. Soulen, C. M. Tuite et al., "Chemoembolization and bland embolization of neuroendocrine tumor metastases to the liver," Journal of Vascular and Interventional Radiology, vol. 18, no. 7, pp. 847-855, 2007.

[19] M. Kalinowski, M. Dressler, A. König et al., "Selective internal radiotherapy with yttrium-90 microspheres for hepatic metastatic neuroendocrine tumors: a prospective single center study," Digestion, vol. 79, no. 3, pp. 137-142, 2009.

[20] A. S. Kennedy, W. A. Dezarn, P. McNeillie et al., "Radioembolization for unresectable neuroendocrine hepatic metastases using resin 90Y-microspheres: early results in 148 patients," American Journal of Clinical Oncology, vol. 31, no. 3, pp. 271279, 2008.

[21] A. Kennedy, W. Dezarn, P. McNeillie et al., "Fractionation, dose selection, and response of hepatic metastases of neuroendocrine tumors after 90Y-microsphere brachytherapy," Brachytherapy, vol. 5, no. 2, p. 103, 2006, Presented as a poster at 2006 Annual American Brachytherapy Society Meeting, abstract P-75.

[22] J. King, R. Quinn, D. M. Glenn et al., "Radioembolization with selective internal radiation microspheres for neuroendocrine liver metastases," Cancer, vol. 113, no. 5, pp. 921-929, 2008.

[23] T. K. Rhee, R. J. Lewandowski, D. M. Liu et al., "90Y radioembolization for metastatic neuroendocrine liver tumors: preliminary results from a multi-institutional experience," Annals of Surgery, vol. 247, no. 6, pp. 1029-1035, 2008.

[24] S.G. Meranze, P. R. Bream, and E. Grzeszczak, "Phase II clinical trial of yttrium-90 resin microspheres for the treatment of metastatic neuroendocrine tumor," in Proceedings of the Society of Interventional Radiology (SIR) 32nd Annual Scientific Meeting, 2007, abstract 422.

[25] S. McGrath, A. Kennedy, and W. Dezarn, "Resin 90Y-microsphere radioembolisation is effective in controlling hepatic metastases from neuroendocrine primary cancers," in Proceedings of the Emerging Trends in Radioembolization Using Microspheres: 3rd Annual Clinical Symposium, May 2007, abstract.

[26] A. Saxena, T. C. Chua, L. Bester, A. Kokandi, and D. L. Morris, "Factors predicting response and survival after yttrium-90 radioembolization of unresectable neuroendocrine tumor liver metastases: a critical appraisal of 48 cases," Annals of Surgery, vol. 251, no. 5, pp. 910-916, 2010.

[27] A. S. Kennedy, P. McNeillie, W. A. Dezarn et al., "Treatment parameters and outcome in 680 treatments of internal radiation with resin 90Y-microspheres for unresectable hepatic tumors," International Journal of Radiation Oncology Biology Physics, vol. 74, no. 5, pp. 1494-1500, 2009.

[28] A. Riaz, R. J. Lewandowski, L. M. Kulik et al., "Complications following radioembolization with yttrium-90 microspheres: a comprehensive literature review," Journal of Vascular and Interventional Radiology, vol. 20, no. 9, pp. 1121-1130, 2009.

[29] R. S. Chamberlain, K. T. Brown, D. Canes et al., "Hepatic neuroendocrine metastases: does intervention alter outcomes?" Journal of the American College of Surgeons, vol. 190, no. 4, pp. 432-445, 2000.

[30] O. Soreide, T. Berstad, A. Bakka et al., "Surgical treatment as a principle in patients with advanced abdominal carcinoid tumors," Surgery, vol. 111, no. 1, pp. 48-54, 1992. 
[31] G. A. Kaltsas, G. M. Besser, and A. B. Grossman, "The diagnosis and medical management of advanced neuroendocrine tumors," Endocrine Reviews, vol. 25, no. 3, pp. 458-511, 2004.

[32] I. M. Modlin, M. Kidd, I. Latich, M. N. Zikusoka, and M. D. Shapiro, "Current status of gastrointestinal carcinoids," Gastroenterology, vol. 128, no. 6, pp. 1717-1751, 2005.

[33] D. A. Osborne, E. E. Zervos, J. Strosberg et al., "Improved outcome with cytoreduction versus embolization for symptomatic hepatic metastases of carcinoid and neuroendocrine tumors," Annals of Surgical Oncology, vol. 13, no. 4, pp. 572$581,2006$.

[34] O. Hauso, B. I. Gustafsson, M. Kidd et al., "Neuroendocrine tumor epidemiology: contrasting Norway and North America," Cancer, vol. 113, no. 10, pp. 2655-2664, 2008.

[35] J. C. Yao, M. Hassan, A. Phan et al., "One hundred years after "carcinoid": epidemiology of and prognostic factors for neuroendocrine tumors in 35,825 cases in the United States," Journal of Clinical Oncology, vol. 26, no. 18, pp. 3063-3072, 2008.

[36] A. Frilling, J. Li, E. Malamutmann, K. W. Schmid, A. Bockisch, and C. E. Broelsch, "Treatment of liver metastases from neuroendocrine tumours in relation to the extent of hepatic disease," British Journal of Surgery, vol. 96, no. 2, pp. 175-184, 2009.

[37] J. G. Touzios, J. M. Kiely, S. C. Pitt et al., "Neuroendocrine hepatic metastases: does aggressive management improve survival?" Annals of Surgery, vol. 241, no. 5, pp. 776-785, 2005. 


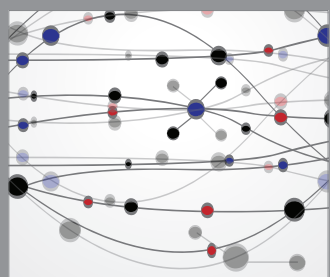

The Scientific World Journal
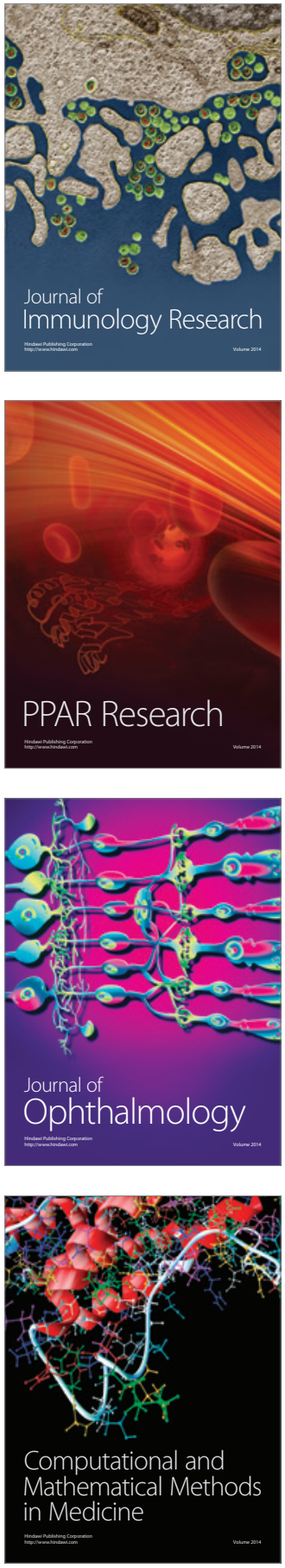

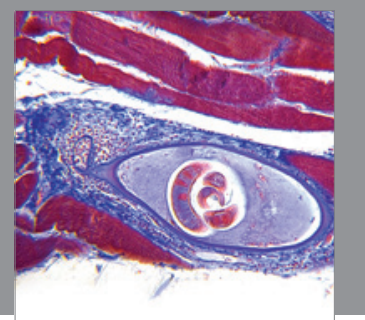

Gastroenterology

Research and Practice
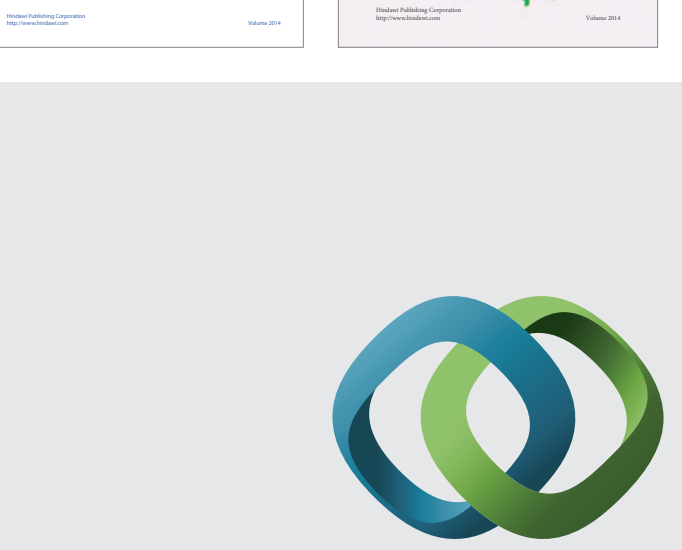

\section{Hindawi}

Submit your manuscripts at

http://www.hindawi.com
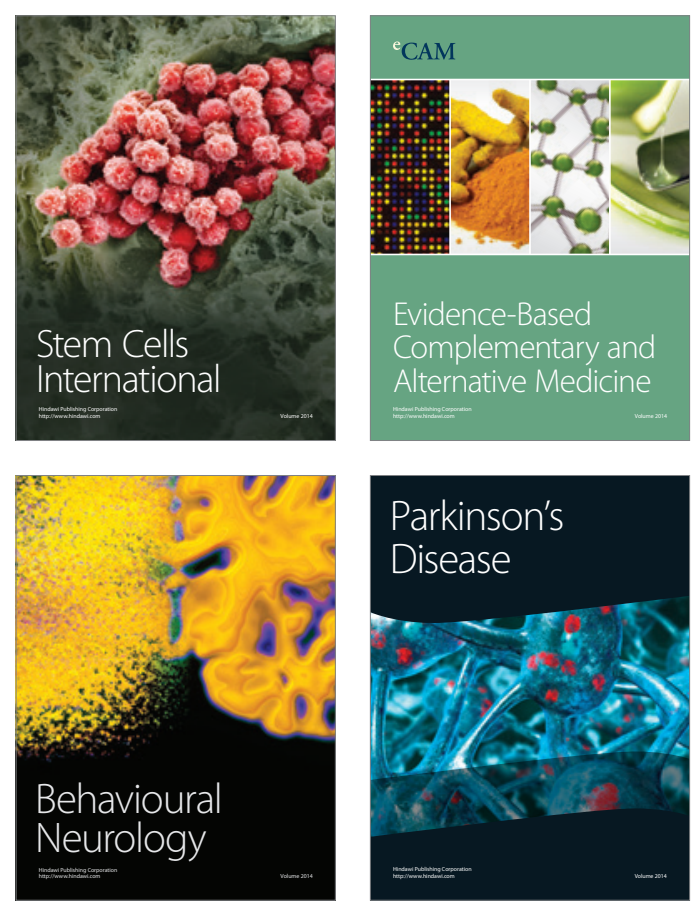

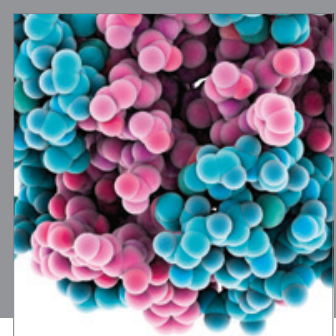

Journal of
Diabetes Research

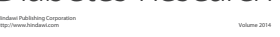

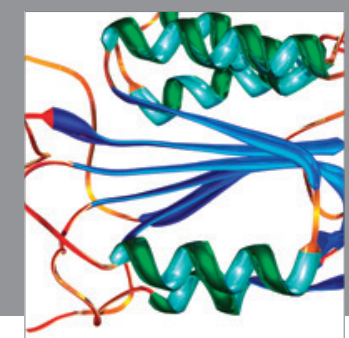

Disease Markers
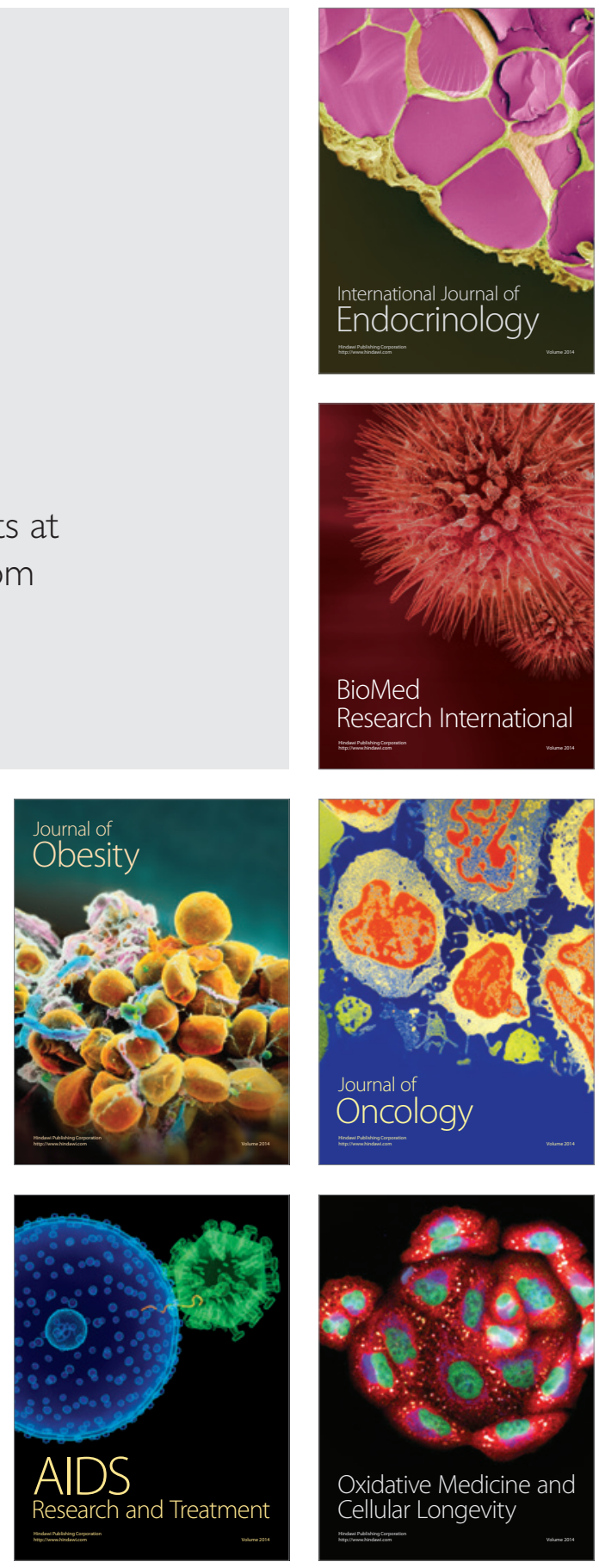